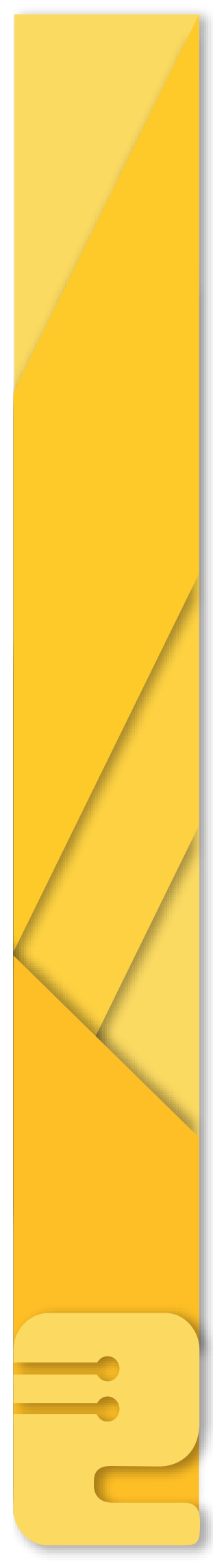

\title{
TERMOELECTRICIDAD: USO DE LAS CELDAS PELTIER EN EL CAMPO DE LA REFRIGERACIÓN Y SUS PRINCIPALES APLICACIONES ${ }^{1}$
}

\author{
THERMOELECTRICITY: \\ USE OF PELTIER CELLS IN THE REFRIGERATION FIELD AND ITS MAIN USES \\ TERMOELECTRICIDADE: \\ USO DAS CELAS PELTIER NO CAMPO DA REFERIGERAÇÃO E SEUS PRINCIPAIS \\ APLICATIVOS \\ Cristian Rubio Ramírez², Guillermo Martheyn Lizarazo³, Emilio Vera Duarte ${ }^{4}$
}

Fecha de recibido: Febrero 15 de 2017 | Fecha de aprobado: Abril 05 de 2017

\section{Resumen}

En este artículo se señala la importancia que han tenido las celdas Peltier en el desarrollo tecnológico e industrial, demostrando, desde un punto de vista científico y matemático, su funcionamiento, los elementos que las componen y el cómo son aprovechadas de forma eficiente mediante el análisis crítico de todos los estudios que tienen como punto focal la termoelectricidad. Se compara el esquema investigativo elaborado hasta la actualidad -correspondiente a las celdas Peltier en el campo de la refrigeración-, organizado por segmentos de texto en los que se explica de manera teórica el funcionamiento y se destaca la producción de algunos modelos matemáticos.

Palabras clave: celdas Peltier, modelos matemáticos, refrigeración, termoelectricidad.

1 Artículo de revisión.

2 Ingeniero mecánico, investigador de la Universidad Francisco de Paula Santander. Pertenece al grupo de investigación Gidpi. Correo electrónico: cristianricardorr@ufps.edu.co.

3 Ingeniero mecánico, investigador de la Universidad Francisco de Paula Santander. Pertenece al grupo de investigación Gidpi. Correo electrónico: guillermoalfredomli@ufps.edu.co.

4 Ingeniero mecánico, magíster en Ciencias de Ingeniería Mecánica, docente investigador de la Universidad Francisco de Paula Santander. Pertenece al grupo de investigación Fluter. Correo electrónico: luisemiliovd@ ufps.edu.co. 


\section{Abstract}

This article points out the importance of the Peltier cells in technological and industrial development. We try to demonstrate, from a scientific and mathematical point of view, its operation, the elements that compose them and how they are efficiently harnessed through critical analysis of all studies that focus on thermoelectricity. We compare the research scheme developed up to now - corresponding to the Peltier cells in the refrigeration field of studies -, organized by segments of text in which its operation is explained in a theoretical way as well as highlighting the production of some mathematical models.

Key Words: Peltier Cells, mathematical models, refrigeration, thermoelectricity.

\section{Resumo}

Neste artigo assinala-se a importância que têm tido as celas Peltier no desenvolvimento tecnológico e industrial, demonstrando, desde um ponto de vista científico e matemático, seu funcionamento, os elementos que as compõem e o como são aproveitadas de forma eficiente mediante a análise crítica de todos os estudos que têm como punto focal la termoelectricidade. Compara-se o esquema investigativo elaborado até a atualidade -correspondente às celas Peltier no campo da referigeração-, organizado por segmentos de texto nos que se explica de maneira teórica o funcionamento e se destaca a produção de alguns modelos matemáticos.

Palavras-chave: celas Peltier, modelos matemáticos, referigeração, termoelectricidade. 


\section{INTRODUCCIÓN}

Las aplicaciones industriales que se observan hoy en día parten del estudio de un principio físico desarrollado anteriormente. Tal es el caso de las celdas Peltier, pequeños dispositivos o células termoeléctricas que producen determinado voltaje a partir de una diferencia de temperatura. En la actualidad, diversos aparatos se destacan por presentar este tipo de sistemas de refrigeración, basados en postulados físicos tales como el efecto Seebeck, el efecto Thomson y el efecto Peltier. No obstante, es preciso aclarar que la celda Peltier es un elemento poco explotado por las empresas que fabrican tecnología electrónica debido a la dificultad de obtener un comportamiento estructurado o, posiblemente, de detallar un patrón de funcionamiento lineal o predecible de este.

Por medio de este artículo se impulsa la búsqueda de nuevas alternativas de generación de energía, permitiendo el acceso a los últimos avances de la industria de las ciencias aplicadas, y así, tomar esto como punto de partida al momento de emprender un proyecto relacionado con tecnología termoeléctrica. El descubrimiento de los fenómenos termoeléctricos ha permitido aumentar la eficiencia en los sistemas donde el desperdicio de calor es considerado, a tal punto que los desarrollos tecnológicos de los últimos treinta años tienen como punto central aprovechar esta energía perdida (Alem, 2009; Díaz Tarasco, 2008).

A escala atómica, considerando una pequeña distribución de masa, un gradiente de temperatura aplicado provoca cargas en el material. Este caso se puede poner en un contexto práctico cuando se calienta un gas clásico que se expande. Parte del volumen que se encuentra a mayor temperatura tiende a desplazarse hacia el lado frío (Bolatti, 2007); a partir de este concepto, se basa todo el campo de la termoelectricidad.

\section{EFECTOS TERMOELÉCTRICOS}

Conocidos como efectos termoeléctricos, estos fenómenos, que involucran la correlación entre corrientes eléctricas y voltajes contra transferencias de calor y temperatura, son los principales artífices de que la termoelectricidad tenga cabida en el mundo de la refrigeración. A continuación, en la tabla 1 , se muestra un cuadro comparativo que consiste en la descripción de cada uno de estos efectos, sus características y esquemas representativos.

Tabla 1. Comparación de efectos termoeléctricos

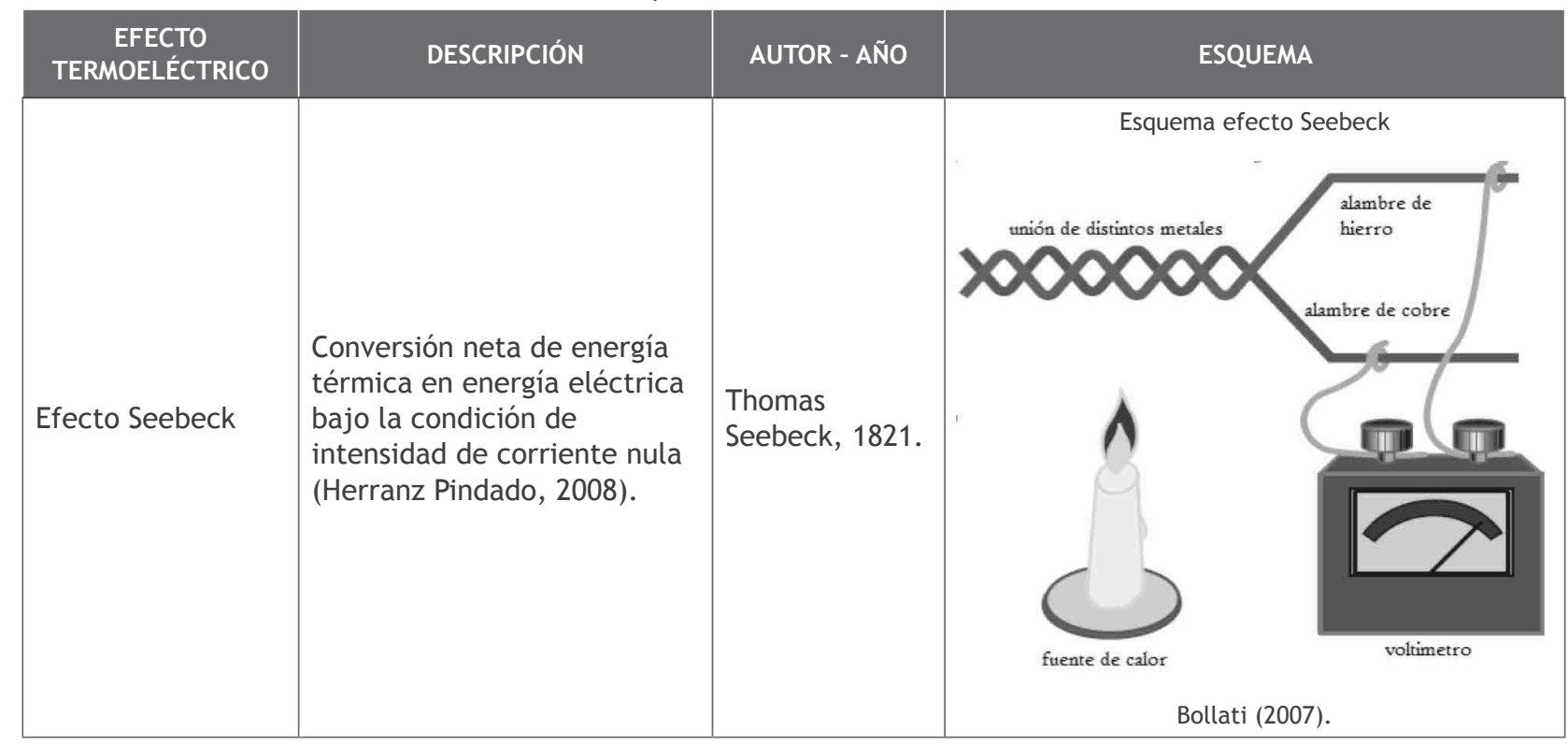




\begin{tabular}{|c|c|c|c|}
\hline $\begin{array}{c}\text { EFECTO } \\
\text { TERMOELÉCTRICO }\end{array}$ & DESCRIPCIÓN & AUTOR - AÑO & ESQUEMA \\
\hline Efecto Peltier & $\begin{array}{l}\text { Enfriamiento o } \\
\text { calentamiento de una unión } \\
\text { entre dos conductores } \\
\text { distintos al pasar una } \\
\text { corriente eléctrica por ella. } \\
\text { Depende de la composición } \\
\text { y temperatura de la unión } \\
\text { (Patterson \& Sobral, 2007). }\end{array}$ & $\begin{array}{l}\text { Jean Peltier, } \\
1834 .\end{array}$ & Msquema efecto Peltier \\
\hline Efecto Thomson & $\begin{array}{l}\text { Absorción o cesión de calor } \\
\text { que tiene lugar cuando una } \\
\text { corriente eléctrica circula } \\
\text { a lo largo de un conductor } \\
\text { que tiene diferentes } \\
\text { temperaturas entre sus } \\
\text { extremos (Díaz Tarasco, } \\
\text { 2008). }\end{array}$ & $\begin{array}{l}\text { William } \\
\text { Thomson, } \\
\text { conocido como } \\
\text { Lord Kelvin, } \\
1851 .\end{array}$ & Esquemas efecto Thomson \\
\hline
\end{tabular}

Fuente: tomado y adaptado de Bollati (2007) y Herranz Pindado (2008).

\section{MODELOS MATEMÁTICOS}

Al momento de analizar un dispositivo termoeléctrico, se deben conocer los fundamentos físicos y matemáticos bajo los cuales estos se rigen. Se mostrarán las ecuaciones que describen los efectos termoeléctricos enunciados (Herranz Pindado, 2008). Es necesario conocer cómo el calor generado, con la diferencia de voltajes, circula a través del conductor; este mecanismo de transferencia de calor es explicado mediante la Ley de Fourier. El efecto Fourier da lugar a un flujo de calor longitudinal -figura 4que aparece en metales de conductividad térmica $K$ en presencia de un gradiente de temperaturas $\nabla T$.

Figura 4. Conducción de calor longitudinal

$$
\mathrm{T}_{2}>\mathrm{T}_{1}
$$

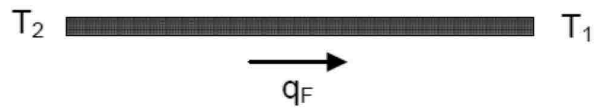

Fuente: Sandoval, Espinosa \& Barahona (2010).

El calor que circula a través del conductor se basa en la Ley de Fourier:

$$
q_{f}=\frac{K}{L} * A_{x} * \Delta T
$$

Donde $K$ es la conductividad térmica propia de cada material, $L$ es la longitud del elemento, $A_{x}$ es el área transversal al flujo de calor y el gradiente $\nabla T$ es la resta entre las temperaturas de los dos extremos, en tales casos, las juntas donde se aplica la corriente eléctrica. Además del calor que se transmite por el efecto Fourier, es menester conocer el calor que se genera por corriente eléctrica. Este fenómeno es conocido como efecto Joule y se enuncia mediante la ecuación:

$$
q_{J}=I^{2} * R
$$

Donde $Q$ es la energía calorífica producida por la corriente, I es la intensidad de la corriente que circula, $R$ es la resistencia eléctrica del conductor. En este caso, observamos que no es considerado el tiempo en la ecuación, pues se considerará el fenómeno dentro de un estado estable.

De otro lado, el efecto Seebeck también es regido por una fórmula matemática. El efecto producido es la aparición de un potencial eléctrico, $V_{0}$, 
proporcional al gradiente térmico impuesto. La constante de proporcionalidad $a_{X Y}$ se denomina coeficiente termoeléctrico dado en $\mathrm{V} / \mathrm{K}$ o $\mathrm{V} /{ }^{\circ} \mathrm{C}$.

$$
V_{o}=\int_{T_{C}}^{T_{h}} \alpha_{X Y} * d T
$$

Donde $T_{h}$ y $T_{c}$ son las temperaturas del extremo caliente y el extreme frío, respectivamente. Sin embargo, como el efecto Peltier es el inverso del efecto Seebeck, el resultado producido es un flujo de calor transversal al conductor no homogéneo, es decir, la unión de metales, y se puede calcular por la ecuación:

$$
q_{p}=-I * T\left(\alpha_{X}-\alpha_{Y}\right)
$$

Donde $T$ es la temperatura conocida, la cual será más alta que la temperatura que se generará al otro lado del semiconductor, además, $a_{x}$ y $a_{y}$ son los coeficientes termoeléctricos de cada metal.

Por último, se identifica el principio bajo el cual actúa el efecto Thomson. El efecto producido es la aparición de un potencial eléctrico, $V_{T}$, proporcional al gradiente térmico impuesto, que da como resultado un flujo calor $q_{T}$ :

$$
\begin{gathered}
V_{t}=-\int_{T_{1}}^{T_{2}} T * \frac{d \alpha}{d T} * d T \\
q_{T}=I * V_{T}
\end{gathered}
$$

Donde $T_{1}$ y $T_{2}$ son las temperaturas de las uniones de los metales.

\section{CELDAS PELTIER}

Una célula o celda Peltier mueve energía calorífica desde la placa fría a la placa caliente a través del control de la energía eléctrica proporcionada por una fuente de alimentación (Dpto. de Electrónica e Informática Industrial, 2007; González García, 2011). Por lo general, una celda Peltier está conformada por dos materiales semiconductores, uno tipo P y otro tipo N, como lo muestra la figura 5. Las células que se comercializan en el mercado esencialmente están compuestas por dos tipos de elementos semiconductores: teluro de bismuto y seleniuro de antimonio (Sandoval, Espinosa \& Barahona, 2010).

La principal ventaja de utilizar celdas Peltier en los procesos de refrigeración es la posibilidad de crear un flujo térmico a partir de una corriente eléctrica de manera directa y eficiente, pues hace inútil el empleo de gases como el neón, que resultan perjudiciales para la capa de ozono (Patterson \& Sobral, 2007).

Figura 5. Componentes de una celda Peltier

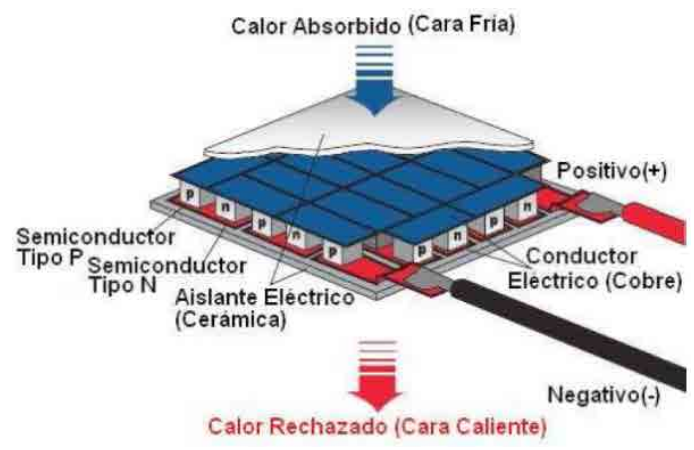

Fuente: Meca Meca \& Jiménez Calvo (2009).

Las placas cerámicas que están dispuestas en ambas caras llevan pistas de cobre que permiten unir los semiconductores eléctricamente en serie y térmicamente en paralelo -ver figura 6-. Para obtener un voltaje útil hace falta una diferencia de temperatura de $100{ }^{\circ} \mathrm{C}$ o más, o conectar muchas células en serie.

Figura 6. Esquemas en serie y paralelo

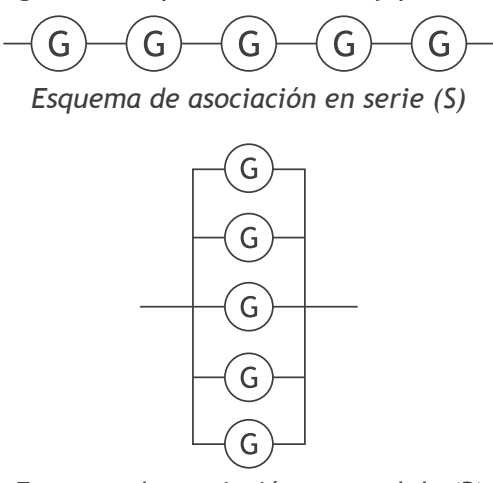

Esquema de asociación en paralelo $(P)$

Fuente: Cid Sarria \& Rodríguez Rebelo (2012).

\section{APLICACIÓN EN REFRIGERACIÓN}

Para seleccionar el equipo que satisfaga las necesidades de refrigeración es necesario conocer inicialmente:

- Temperatura de diseño del medio ambiente.

- Requerimientos de temperatura y humedad del producto almacenado.

- Dimensiones, tipo de construcción, aislamiento y exposición solar, si la hay, del cuarto frío.

- Clase del producto, cantidad o peso y clase de operación. 
- Servicio eléctrico, iluminación, equipos, manejo del producto dentro del cuarto frío.

- Capacidad -potencia- de los motores de evaporadores y calor de descongelación (Granados Granados, 2011; Alem, 2009).

Físicamente, los elementos de un módulo Peltier son bloques de $1 \mathrm{~mm}^{3}$ conectados eléctricamente en serie y térmicamente en paralelo. Tenemos en cuenta sus reducidas dimensiones - de unos milímetros escasos-, una sola célula puede alcanzar como máximo una potencia frigorífica de 0,5 W. (Iniesta \& Pérez, 2002). Es decir, para conseguir potencias frigoríficas de 15 a 20 W hay que realizar baterías formadas, como mínimo por treinta o cuarenta células. De hecho, al aumentar el número de células, aumenta la superficie irradiante $y$, por tanto, la potencia refrigerante -ver figura 7-.
Figura 7. Arreglo de celdas Peltier

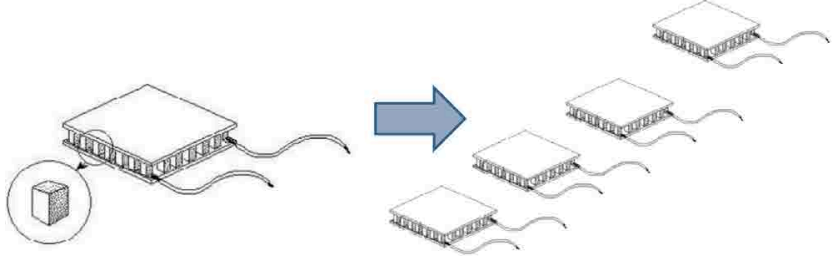

Fuente: Passamai \& Bernaski (2005).

El desarrollo de las celdas Peltier ha permitido fabricar dispositivos capaces de disipar más de $100 \mathrm{~W}$ de calor y obtener una diferencia de temperatura entre sus caras de hasta $70{ }^{\circ} \mathrm{C}$, como lo muestra la figura 8.

Figura 8. Montaje de sistema de refrigeración usando celdas Peltier
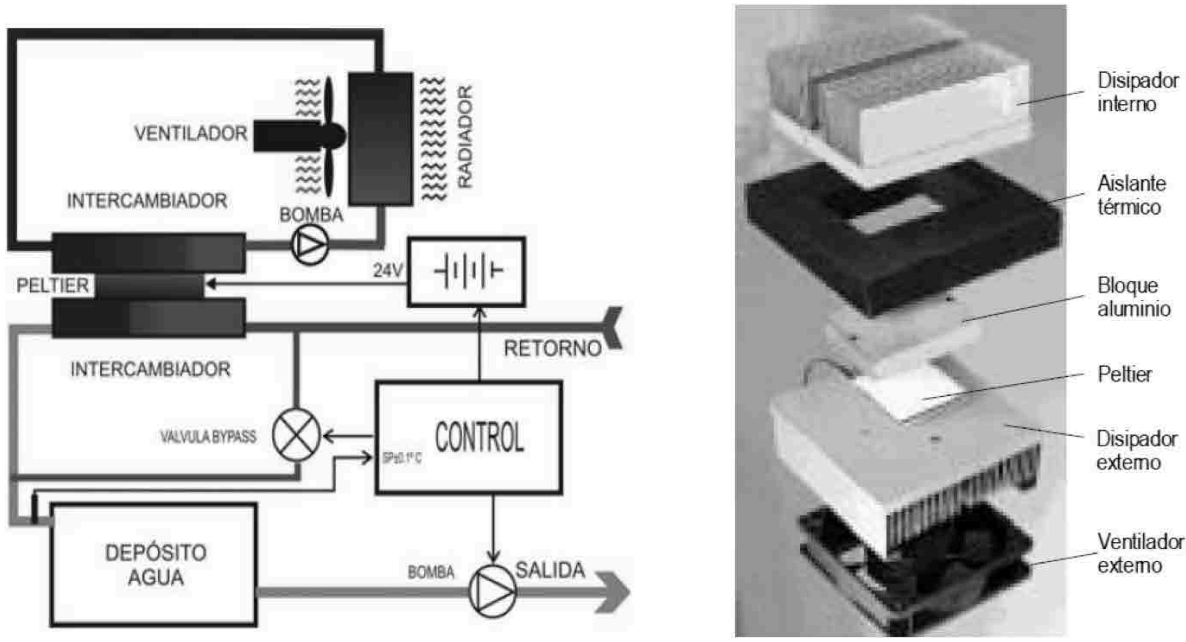

Fuente: González García (2011); Meca Meca \& Jiménez Calvo (2009).

Las máquinas equipadas con esta tecnología permiten controlar la humedad en climas hostiles de forma eficaz, pues se ha logrado eliminar ruidos y vibraciones, evitando las partes móviles de los compresores que llevan los actuales deshumidificadores y los fluidos frigoríferos.

\section{OTRAS APLICACIONES}

Las compañías de automóviles alemanas Volkswagen у вмш han desarrollado generadores termoeléctriCOS - GTE- que recuperan el gasto de calor de una máquina de combustión. Volkswagen afirma que genera $600 \mathrm{~W}$ de salida del generador termoeléctrico en condición de conducción en autopista. La electricidad producida por el GTE es cerca del $30 \%$ de la electricidad requerida por el auto, obteniendo una carga mecánica reducida mediante el alternador de voltajes y una reducción en el consumo de combustible de más del $5 \%$. BMW y DLR - del alemán Deutsches Zentrum für Luft- und Raumfahrt, que en castellano significa Centro Aeroespacial Alemán- han desarrollado también un generador termoeléctrico, impulsado por el tubo de escape, que alcanza un máximo de $200 \mathrm{~W}$ y se ha utilizado exitosamente por más 12000 km de uso en carretera (Díaz Tarasco, 2008).

Los sistemas de enfriamiento de las cámaras digitales tienen pequeños sistemas CCD -sensores con células fotovoltaicas- que funcionan con base en el efecto Peltier, como en el termociclador usado en biología molecular para realizar la PCR, método por el cual se amplifica un fragmento de ADN (Alem, 2009). 
Tabla 2. Síntesis de las últimas aplicaciones de la termoelectricidad

\begin{tabular}{|c|c|}
\hline $\begin{array}{l}\text { ÁMBITO DE } \\
\text { APLICACIÓN }\end{array}$ & EQUIPOS TERMOELÉCTRICOS \\
\hline $\begin{array}{l}\text { Equipos de } \\
\text { laboratorio y } \\
\text { científicos }\end{array}$ & $\begin{array}{l}\text { Diodos láser, placas de control de tempe- } \\
\text { ratura, cámaras de proceso y climáticas, } \\
\text { baños de referencia de punto de conge- } \\
\text { lación, baños de temperatura constante, } \\
\text { higrómetros de punto de rocío, osmóme- } \\
\text { tros, etapas del microscopio. }\end{array}$ \\
\hline $\begin{array}{l}\text { Tecnología } \\
\text { del transporte }\end{array}$ & $\begin{array}{l}\text { Cajas móviles, cabinas o contenedores } \\
\text { para distribución de comida, equipos } \\
\text { médicos, farmacéuticos, por tierra, mar } \\
\text { o aire. }\end{array}$ \\
\hline Medicina & $\begin{array}{l}\text { Almacenamiento móvil o estacionario de } \\
\text { sangre o farmacéuticas, instrumentos, } \\
\text { mantas de hipotermia, enfriadores, con- } \\
\text { geladores de córnea oftálmica, analiza- } \\
\text { dores de sangre, preparación y almace- } \\
\text { naje de tejidos. }\end{array}$ \\
\hline $\begin{array}{l}\text { Militar y } \\
\text { aeroespacial }\end{array}$ & $\begin{array}{l}\text { Cajas portátiles de temperatura cons- } \\
\text { tante para distribución de sangre y sumi- } \\
\text { nistros, dispositivos electrónicos, siste- } \\
\text { mas de orientación inerciales de enfria- } \\
\text { miento y calentamiento, amplificadores } \\
\text { paramétricos y otros equipos en barcos, } \\
\text { submarinos, camiones, y aviones. }\end{array}$ \\
\hline
\end{tabular}

Fuente: adaptación de los autores.

\section{CONCLUSIONES}

Nuevas tecnologías, que se han desarrollado con el paso del tiempo, tienen como objetivo aumentar la eficiencia energética sin afectar de forma radical el medio ambiente. Los efectos termoeléctricos responden a la necesidad de aprovechar el calor que se desperdicia en los sistemas de combustión, la energía eléctrica que podría ser producida gracias a la diferencia de temperatura que se genera en los entornos de trabajos industriales.

Los principales efectos termoeléctricos que rigen los sistemas de este tipo son los siguientes: el efecto Seebeck, con el que por medio de la aplicación de calor y frío a una placa podemos conseguir una intensidad y una diferencia de potencial eléctrico; el efecto Peltier, mediante el cual una determinada corriente eléctrica, que toma una cara caliente, torna su cara opuesta en fría; y el efecto Thomson, en el que, dependiendo de la polarización de la corriente, un conjunto de caras absorberá o generará calor.
El desarrollo de tecnología termoeléctrica presenta un tema de gran interés para el desarrollo de experimentos en laboratorios de electrónica. Su capacidad para enfriar no es muy elevada, pero con las modificaciones pertinentes, desde el punto de vista de control matemático y asistido por software, es previsible una mejora substancial del rendimiento.

En este trabajo se abordó la caracterización de las celdas Peltier y cómo estas ayudan a módulos con los elementos de disipación de calor. La velocidad de respuesta de una celda Peltier es considerablemente alta en comparación con la velocidad de respuesta de sistemas térmicos tradicionales -resistencias calefactoras, bombas de calor, complejos sistemas de aire acondicionado, etc.-; por ello, se piensa que es factible emplear este tipo de elementos como una forma alternativa en aplicaciones relacionadas con la refrigeración.

\section{REFERENCIAS}

Alem, L. (2009). Principios básicos de termoelectricidad. Sistelec, 18. Buenos Aires.

Bollati, E. (2007). Generadores termoeléctricos. Generación de energía sin partes móviles. EE.UU: Corporex S.A./Global Thermoelectric.

Cid Sarria, S., \& Rodríguez Rebelo, F. (2012). Aplicación de las placas Peltier a la generación de energía eléctrica en plataformas flotantes. Ourense, Brasil: Parque Tecnológico de Galicia. Aulas Tecnópole.

Díaz tarasco, D. (2008). Efecto termoeléctrico. Descripción y aplicación. Miranda, Venezuela: Universidad Simón Bolivar.

Dpto. de Electronica e Informática Industrial. (2007). Práctica de Servosistemas. Modelado de una célula Peltier. Escuela Universitaria de Ingeniería Técnica Industrial. Madrid: Universidad Politécnica de Madrid.

González García, J. (2011). Sistema de refrigeración de estado sólido para instrumentación científica. Tenerife, España: Universidad de La Laguna.

Granados Granados, J. (2011). Manual de ventilación, refrigeración y aire acondicionado. Cúcuta: UFPS. 
Granados, J., Fernández, J., García, J., \& Tavera, F. (2008). Caracterización termodinámica de celdas Peltier. XXIII Congreso de Instrumentación Somim. Universidad Autónoma Metropolitana Azcapotzalco. Laboratorio de Óptica, Departamento de Ciencias Básicas, México.

Herranz Pindado, R. (2008). Climatización mediante células Peltier. España: Universidad Pontificia Comillas / Escuela Técnica Superior de Ingeniería.

Iniesta, J., \& Perez, C. (2002). Módulo cerámico miniatura. Series FC- FC. Materials Electronic Products Corporation (Frigichip). EE.UU: Melcor.

Martínez Ordóñez, E., \& Marín, E. (2008). Controlador de temperatura mediante celdas Peltier para detección de transiciones de fase. I Simposio de Tecnología Avanzada. Instituto Politécnico Nacional. Centro de Investigación en Ciencia Aplicada y Tecnología Avanzada, México D.F.

Meca Meca, F., \& Jiménez Calvo, J. (2009). Horno-nevera basado en células Peltier para el ensayo térmico de dispositivos electrónicos. España: Escuela Politécnica Universidad de Alcalá.

Mendoza Razo, A., \& Amaro Betancourt, C. (2010). Efecto Peltier aplicado en una unidad para equipo de medición. xvı Congreso Internacional Anual de la Somim. Instituto Tecnológico de San Luis Potosí. Unidad Tecnológica Soledad de Graciano Sánchez, San Luis de Potosí.

Passamai, V., \& Bernaski, M. (2005). Experiencias de refrigeración con una caja de heladera. Salta, Argentina: Inenco /Ciunsa / Conicet (Facultad de Ciencias Exactas).
Patterson, G., \& Sobral, M. (2007). Efecto Peltier. Dpto. de Física - FCEYN - UBA.

Pérez Salgado, E. (2006). Diseño de un sistema de aire acondicionado portátil termoeléctrico. Cataluña: Universidad Politécnica de Cataluña / Escuela Politécnica Superior de Castelldefels.

Pontigo Vásquez, L. (2011). Rediseño y construcción de un termo-estimulador. Valdivia, Chile: Universidad Austral de Chile / Escuela de Ingeniería Civil Electrónica.

Sacristán Gómez, A. (2011). Obtención de agua a partir de humedad atmosférica empleando células de efecto Peltier. Madrid, España: Universidad Carlos III de Madrid / Escuela Politécnica Superior.

Sandoval, A., Espinosa, E., \& Barahona, J. (2010). Construcción de sistemas de enfriamiento con base en semiconductores. Huajuapan de León, México: Universidad Tecnológica de la Mixteca / Instituto de Electrónica.

Triano, J., Chapino, V., Gareis, G., \& Gauto, P. (2010). Efecto Peltier - Refrigeración con materiales de estado sólido. Regional Paraná, Brasil: Universidad Tecnológica Nacional / Cátedra de Tecnología Electrónica.

Ulloa Solano, N. (2013). Control por hardware de sistemas de gobierno para diodos láser con STM $32 f 4$ y celdas Peltier. Costa Rica: Universidad de Costa Rica / Escuela de Ingeniería Eléctrica. 